\title{
Article \\ Study on Film Cooling Performance of Round Hole Embedded in Different Shaped Craters and Trenches
}

\author{
Xiaojun $\mathrm{Wu}^{1}$, Xin $\mathrm{Du}^{1}$ and Chunhua Wang ${ }^{1,2, *}$ \\ 1 AECC Shenyang Engine Research Institute, No. 1 Wanlian Road, Shenyang 110015, China; \\ 18002492948@163.com (X.W.); dxknight@163.com (X.D.) \\ 2 College of Energy and Power Engineering, Nanjing University of Aeronautics and Astronautics, \\ Nanjing 210016, China \\ * Correspondence: chunhuawang@nuaa.edu.cn
}

Citation: $\mathrm{Wu}, \mathrm{X}$.; Du, X.; Wang, C. Study on Film Cooling Performance of Round Hole Embedded in Different Shaped Craters and Trenches. Aerospace 2021, 8, 147. https://doi.org/10.3390/ aerospace 8060147

Academic Editor: Qiang Zhang

Received: 16 April 2021

Accepted: 17 May 2021

Published: 25 May 2021

Publisher's Note: MDPI stays neutral with regard to jurisdictional claims in published maps and institutional affiliations.

Copyright: (C) 2021 by the authors. Licensee MDPI, Basel, Switzerland. This article is an open access article distributed under the terms and conditions of the Creative Commons Attribution (CC BY) license (https:/ / creativecommons.org/licenses/by/ $4.0 /)$.

\begin{abstract}
Film cooling effectiveness can be improved significantly by embedding a round hole in trenches or craters. In this study, film cooling performances of a transverse trench, $\mathrm{W}$-shaped trench and elliptic trench were compared and analyzed in detail. The CFD models for trench film cooling were established and validated via the experimental results. Inside the transverse trench, a pair of recirculating vortices is formed, which promotes the coolant spreading in a lateral direction. The decrease of trench width and increase of trench depth both improve the film cooling effectiveness of the transverse trench. For the W-shaped trench, the guide effect of the corner angle further improves the lateral spreading capability of coolant and generates higher cooling effectiveness than a transverse trench with the same depth and width. The flow characteristics of the elliptic trench are similar to that of the round hole, and the kidney vortex pair takes a dominant role in the flow fields downstream of the coolant exit. Accordingly, the elliptic trench generates the worst cooling performance in these shaped trenches. The increase of trench depth and decrease of trench width both result in an increase of the discharge coefficient for trench film cooling. For the W-shaped trench, the increase of the corner angle causes a decrease of the discharge coefficient. For the elliptic trench, the discharge coefficient increases with the decrease of the elliptic aspect ratio (major axis/minor axis).
\end{abstract}

Keywords: film cooling; shaped trench; CFD; adiabatic film cooling effectiveness; discharge coefficient

\section{Introduction}

To increase output efficiency, gas turbines are usually operated at the temperature higher than the maximum allowable value for materials. To avoid the thermal damage of the airfoil, various cooling strategies such as film cooling, impingement cooling and pin fins are applied. In the external film cooling process, coolant air extracted from the compressor is ejected through inclined holes and forms a coolant film on the airfoil surface [1]. The coolant film not only cools the airfoil surface, but also reduces the heat flow from the mainstream to hot-section surface. However, for a traditional inclined cylindrical hole, the coolant injection tends to separate from the downstream wall, especially at high jet momentum, and the spanwise coverage of the coolant is also weak. This causes low area-averaged film cooling effectiveness [2,3].

To improve the spanwise coverage of coolant, one approach is to change the hole geometry from round to shaped outlet $[4,5]$. The transition from cylindrical inlet to shaped outlet results in a decrease of outlet coolant momentum. Flow deceleration in the hole diffuser section also promotes the spanwise spreading of coolant, which improves cooling performance. Accordingly, to get the same cooling effectiveness, the coolant consumption decreases. Over the past 40+ years, many different outlet shapes for film cooling holes have been proposed [2,3]. However, because of the limitation of manufacturing conditions, shaped hole technology has not been widely used in practice. 
A certain configuration by embedding a round hole in a transverse trench generates similar cooling performance to shaped holes. As trench configuration can be fabricated via thermal barrier coating (TBC), trench film cooling is more practical compared with other shaped holes [6]. Moreover, transverse trenches with the compound angle injection of a round hole or shaped hole can further improve film cooling performance $[7,8]$. A trench study on a flat plate was performed by Bunker [9]. He reported that, at high jet momentum, the coolant can still attach closely to the surface. This result is confirmed by Harrison and Bogard [10] and Lu et al. [11-13]. The CFD and experimental results from Lu et al. [11-13] showed that, compared with a round hole, higher film effectiveness and heat flux reduction can be generated by embedding a round hole in a trench. Moreover, the heat transfer coefficient does not change obviously after introducing a trench. Trench film cooling on the vane surface was studied by Waye and Board [14]. As the trench width decreases, the cooling effectiveness increases. Even at high jet momentum, the mainstream can suppress the coolant jet ejected from the trench on the vane surface effectively. The influences of trench geometries on temperature distribution on a TBC coated vane were investigated by Davidson et al. [15]. Their results showed that, compared with round holes, trenches and craters can generate much better coolant coverage, however, temperature at the interface of the vane and TBC only shows a slight decrease. Lee and Kim [16] also conducted parametric studies on trench film cooling. A trench height of 1 diameter and trench width of 2 diameter of the round hole generates the highest cooling effectiveness both at low and high jet momentums. Moreover, reverse injection of coolant can improve the film cooling performance.

Besides transverse trenches, some other shaped trenches are also proposed and have been proved to generate higher film cooling effectiveness than traditional round holes [17]. Lu et al. [18] tested film the cooling effectiveness of a round hole embedded in the elliptic crater. Their experimental results show that, compared with round holes, crater film cooling generates a higher heat transfer coefficient and cooling effectiveness. Dorrington et al. [19] also concluded the crater hole generates lower cooling effectiveness than the trench configuration, but higher effectiveness than cylindrical holes. Kross and Pfitzner [20,21] found that placing a tetrahedral element upstream of the trench can improve the cooling performance by reducing the coolant-mainstream mixture within the trench and improving lateral coolant spreading. Wei et al. [22] and Zhang et al. [23] developed double- and sine-wave trenches, and the influences of wave geometries on cooling effectiveness were studied.

In the present study, a systematic parametric study is performed for trench or crater film cooling. CFD models for a transverse trench, $\mathrm{W}$-shaped trench and elliptic trench were established and validated using the experimental results. The flow mechanisms and cooling performances with different trench shapes were analyzed in detail.

\section{Computational Model}

\subsection{Computational Domain}

As illustrated in Figure 1, the computational domain consists of a mainstream channel, coolant channel, cylindrical hole and shaped trench. The cylindrical hole has an inclination angle of $30^{\circ}$ and diameter of $5.0 \mathrm{~mm}$. The total height including the cylindrical section and shaped trench, $h_{\mathrm{t}}$, is $3 d$. The width of the mainstream and coolant channel is $3.0 d$. The coordinate origin locates at the trench exit center, and the $\mathrm{x}, \mathrm{y}$ and $\mathrm{z}$ axes correspond to streamwise, spanwise and vertical direction, respectively. Three kinds of shaped trenches, a transverse trench, $\mathrm{W}$-shaped trench and elliptic trench, were investigated. The geometries of the trench such as depth $(h)$, width $(w)$, corner angle $(\alpha)$ and axis length $\left(D_{\mathrm{x}}, D_{\mathrm{y}}\right)$ are defined in Figure 1. The changing interval of these parameters are listed in Table 1. 


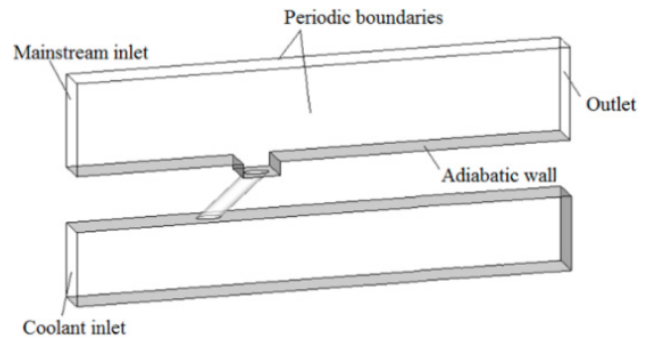

(a) 3D-view
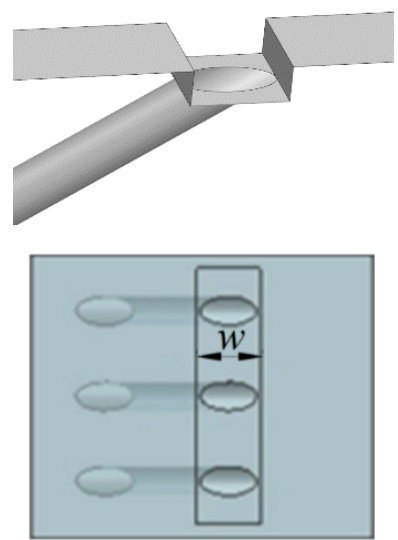

(c) Transverse trench
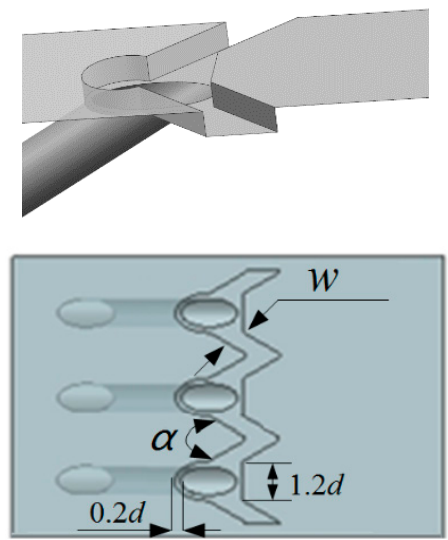

(d) W-shaped trench

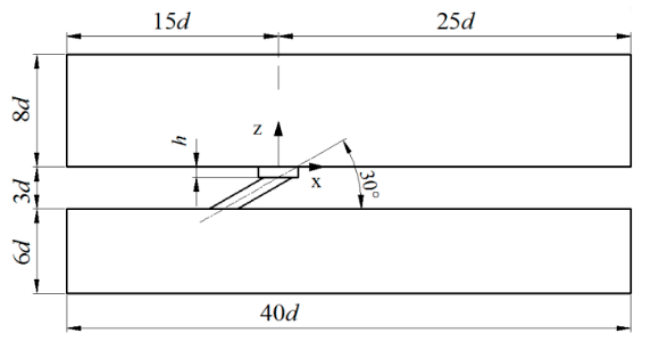

(b) Front view

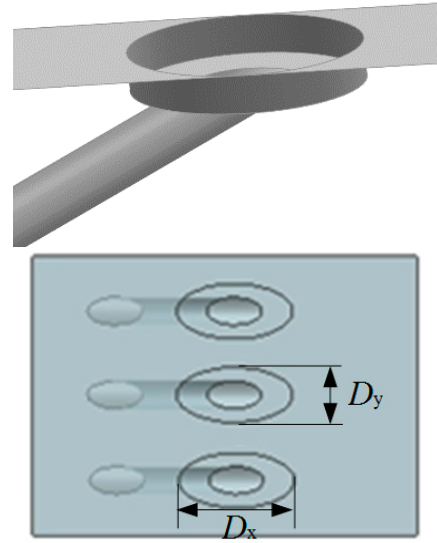

(e) Elliptic trench

Figure 1. Computational domain and geometric variables for trench holes in the present study.

Table 1. Changing interval of trench geometries.

\begin{tabular}{ccc}
\hline Trench Type & Symbol & Changing Interval \\
\hline \multirow{2}{*}{ Transverse trench } & $W$ & $2.5 \sim 3.5 d$ \\
& $h$ & $0.25 \sim 1.8 d$ \\
\hline \multirow{3}{*}{ W-shaped trench } & $W$ & $1.2 \sim 2.4 d$ \\
& $h$ & $0.25 \sim 1.8 d$ \\
Elliptic trench & $\alpha$ & $40 \sim 80^{\circ}$ \\
& $D_{\mathrm{x}}$ & $1.2 \sim 3.2 d$ \\
& $D_{\mathrm{y}}$ & $1.2 \sim 3.2 d$ \\
& $h$ & $0.25 \sim 1.8 d$ \\
\hline
\end{tabular}

\subsection{Performance Evaluation Parameters for Film Cooling}

Adiabatic cooling effectiveness is an important index to evaluate film cooling performance, and can be calculated by:

$$
\begin{gathered}
\eta_{\mathrm{ad}, \mathrm{loc}}(x, y)=\frac{T_{\infty}-T_{\mathrm{ad}, \mathrm{w}}}{T_{\infty}-T_{\mathrm{c}}} \\
\eta_{\mathrm{ad}, \text { lat }}(x)=\frac{1}{\Delta y} \int_{-\Delta y / 2}^{\Delta y / 2} \eta_{\mathrm{ad}, \text { loc }}(x, y) \mathrm{d} y \\
\eta_{\mathrm{ad}, \mathrm{av}}=\frac{1}{\Delta x} \int_{x_{1}}^{x_{2}} \eta_{\mathrm{ad}, \text { lat }}(x) \mathrm{d} x
\end{gathered}
$$

where $T_{\mathrm{ad}, \mathrm{w}}$ is the adiabatic wall temperature, and $T_{\mathrm{c}}$ and $T_{\infty}$ are the temperature of the coolant and mainstream. The subscripts ' $\mathrm{loc}^{\prime}$, 'lat' and 'av' denote the local, laterally 
averaged and area-averaged value, respectively. In the present study, $\triangle y=3 d, x_{1}=2 d$ and $x_{2}=20 d$. The discharge coefficient, $C_{\mathrm{d}}$, is defined as

$$
C_{\mathrm{d}}=\frac{m_{\mathrm{c}}}{A_{\mathrm{c}} \sqrt{2 \rho_{\mathrm{c}}\left(P^{*}{ }_{\mathrm{c}, \text { in }}-P_{\mathrm{c}, \text { out }}\right)}}
$$

where $m_{\mathrm{c}}$ is the coolant mass, $A_{\mathrm{c}}$ is the cross section area of the round hole, $P^{*} \mathrm{C}$,in and $P_{\mathrm{c}, \mathrm{out}}$ are the inlet total pressure of secondary flow and the static pressure downstream of the trench.

\subsection{Boundary Condition and Solution Method}

At the mainstream inlet, the velocity profile with a $1 / 7$ power law and the boundary thickness $\left(\delta_{99}\right)$ of $0.125 d$ were specified. This is the same as that in the experimental conditions. The mainstream mean velocity is $20 \mathrm{~m} / \mathrm{s}$, and the temperature is $353 \mathrm{~K}$. The turbulent intensity and length scale is $4 \%$ and $0.4 \delta_{99}$ respectively. The coolant temperature is $300 \mathrm{~K}$. The turbulent parameters at the coolant inlet are the same as the mainstream inlet. Because of a low Mach number $(<0.3)$, incompressible ideal gas is used. The top surface of the mainstream channel was set as a free boundary. The spanwise surfaces of the mainstream and coolant channel were set as periodic boundaries. Other surfaces were set as a non-slip adiabatic wall. In the present study, the density ratio is 1.176, the blowing ratio $\left(M=\rho_{\mathrm{c}} u_{\mathrm{c}} / \rho_{\infty} u_{\infty}\right)$ is $0.5 \sim 3.0$ and the momentum ratio $\left(I=\rho_{\mathrm{c}} u_{\mathrm{c}}{ }^{2} / \rho_{\infty} u_{\infty}{ }^{2}\right)$ is 0.21 7.65.

ANSYS Fluent is applied for solutions of governing equations. According to Ref. [10], realizable $k-\varepsilon$ equations with enhanced wall treatment are suitable for trench film cooling. The momentum, energy and turbulent equations were solved using a second-order upwind scheme. The gradient and pressure interpolation were performed using a least squares cell-based scheme and second-order scheme, respectively. The convergence criteria include: (1) the mass balance error is smaller than $10^{-6}$, (2) the normalized residuals are smaller than $10^{-6}$, and (3) the variation of local adiabatic effectiveness is smaller than $10^{-2}$.

Structural meshes are created with ICEM software. As shown in Figure 2, near the walls, the grid points are clustered. On the flat plate, the wall-normal size of the firstlayer grid is $\triangle z=0.003 d$, which corresponds to $z^{+} \approx 1$. In the wall-normal direction, the stretching factor is smaller than 1.2 in the near-wall region. Grid independence tests were carried out to determine the optimal grid number. Taking the transverse trench with $w=2.2 d$ and $h=0.5 d$ as an example, the grid test result is shown in Figure 3. The calculation results do not change obviously as the grid number exceeds $1,896,323$.

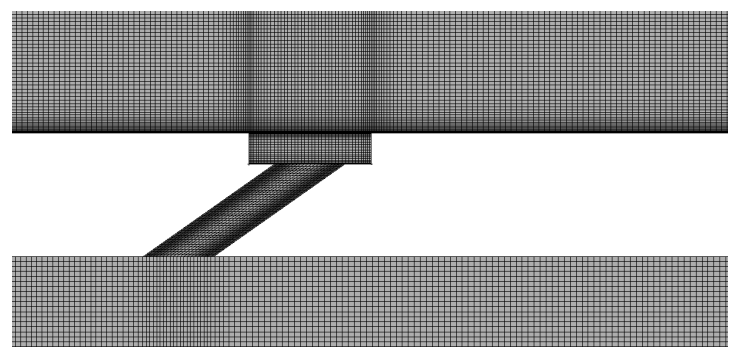

(a) Front view

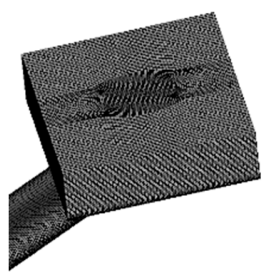

(b) Transverse trench

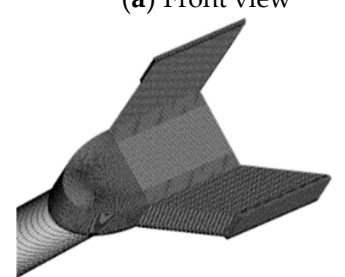

(c) W-shaped trench

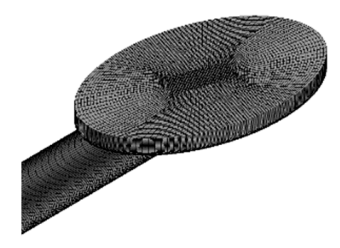

(d) Elliptic trench

Figure 2. Grids used in the present study. 


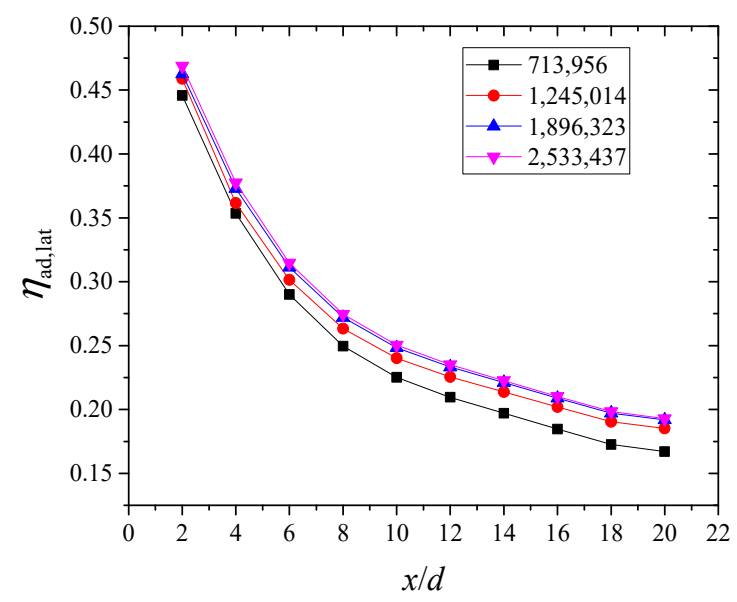

Figure 3. Gird independent test results.

\section{Experimental Validation}

Figure 4 shows the experimental system. After being heated to $80^{\circ} \mathrm{C}$, the mainstream from an air compressor passes through a rectifier section and then enters the test sections. The cross section size of the mainstream channel is $174 \mathrm{~mm} \times 80 \mathrm{~mm}$, and the size of the coolant channel is $64 \mathrm{~mm} \times 40 \mathrm{~mm}$. The inlet velocity and temperature are the same as the CFD model. The boundary layer thickness and turbulent parameters for the mainstream inlet were measured at $x / d=-15$ using a hot wire anemometer (StreamLine Pro). The boundary layer thickness $\left(\delta_{99}\right)$ is $0.125 d$. The turbulent intensity and length scale is $4 \%$ and $0.4 \delta_{99}$. The test plate, with a thermal conductivity of $0.3 \mathrm{~W} /(\mathrm{m} \cdot \mathrm{K})$, is made of rubber wood. The sizes of the hole and the trench are the same as the computational model. The hole pitch is $3.0 d$. The transverse trench $(w=3.2 d, h=1 d)$, the W-shaped trench $\left(w=1.7 d, h=1 d\right.$ and $\left.\alpha=60^{\circ}\right)$ and the elliptic trench $\left(D_{\mathrm{x}}=2.2 d, D_{\mathrm{y}}=2.8 d\right.$ and $\left.h=1 d\right)$ are tested in the present study. Viewing though $\mathrm{CaF}_{2}$-infrared glass, an infrared thermography system is applied for temperature measurement of the flat plate with black paint coating. The emissivity of the black paint is about 0.97. The infrared thermography (Mag32HF model) produced by Magnity Electronics Co. Ltd. (Shanghai, China) has a test range of $-20 \sim 300{ }^{\circ} \mathrm{C}$ and an accuracy of $\pm 1{ }^{\circ} \mathrm{C}$. The calibration of the infrared measurement was performed according to the temperature measured via thermocouples within the plate. Detail calibration processes are introduced in Ref. [24].

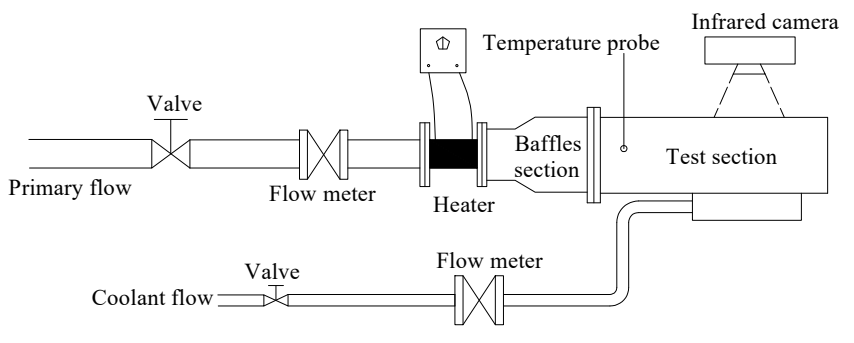

(a) Experimental system

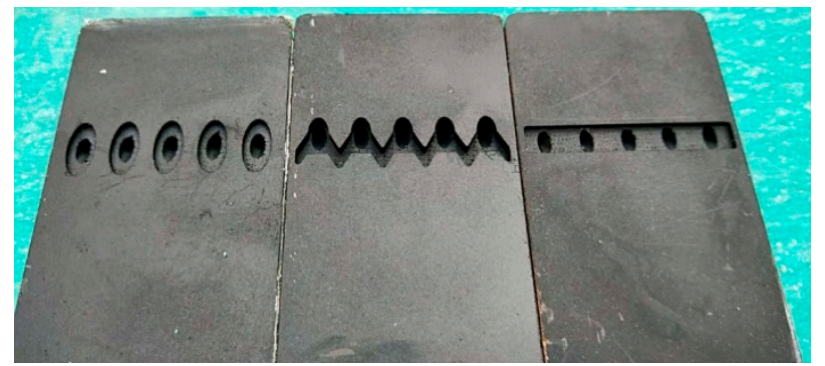

(b) Test plates

Figure 4. Experimental system in the present study.

To better compare the CFD and experimental results, the heat conduction effect inside the film-cooling plate is taken into account, and the thermal conductivity is $0.3 \mathrm{~W} /(\mathrm{m} \cdot \mathrm{K})$. The top and lower surfaces of the solid plate were coupled with the fluid phase. The spanwise surfaces of the plate were set as periodic boundaries. Figure 5 shows the distributions of the overall cooling effectiveness $\left[\eta_{\text {overall }}=\left(T_{\infty}-T_{\mathrm{w}}\right) /\left(T_{\infty}-T_{\mathrm{c}}\right)\right]$ on the cooling surface. Inside the trench, the difference between the CFD and the experimental results is somewhat 
obvious, especially for the transverse trench at a high blowing ratio. It illustrates that the present CFD model overestimated the spreading capability of coolant inside the trench, which results in better cooling performance. Figure 6 shows a quantitative comparison between the experimental and CFD results. The experimental data for the round hole is from Ref. [25]. At $M=0.5$, the mean relative error for the round hole, transverse trench, $\mathrm{W}$-shaped trench and elliptic trench is about $9.4 \%, 7.7 \%, 8.5 \%$ and $10.6 \%$. At $M=1.5$, the mean relative error for the round hole, transverse trench, $\mathrm{W}$-shaped trench and elliptic trench is about $16.4 \%, 10.2 \%, 12.2 \%$ and $13.9 \%$. Overall, the CFD results agree well with the experimental results.

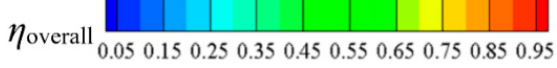

$\operatorname{Exp}$

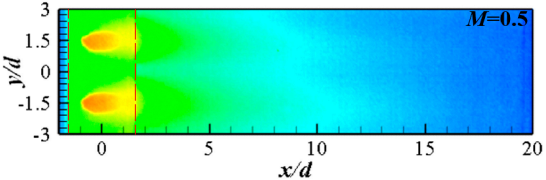

CFD
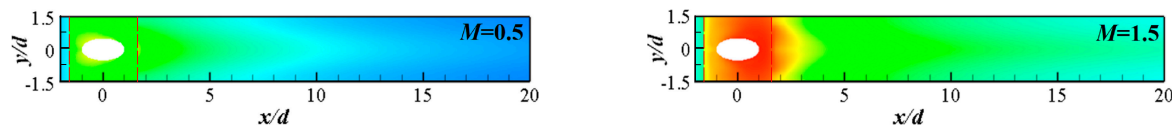

(a) Transverse trench $(w=3.0 d, h=1.0 d)$

Exp
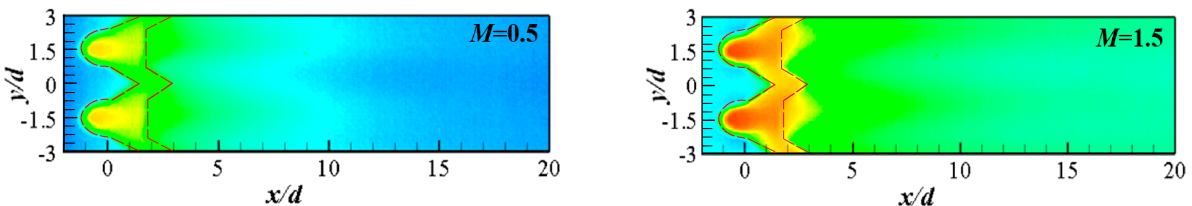

CFD
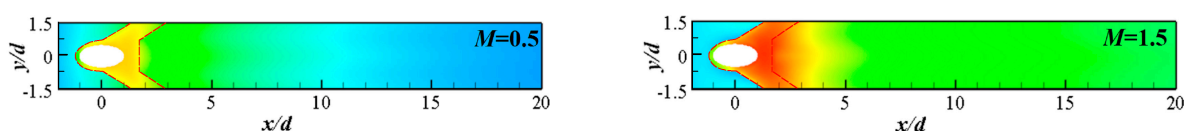

(b) W-shaped trench $\left(w=1.7 d, h=1 d, \alpha=60^{\circ}\right)$
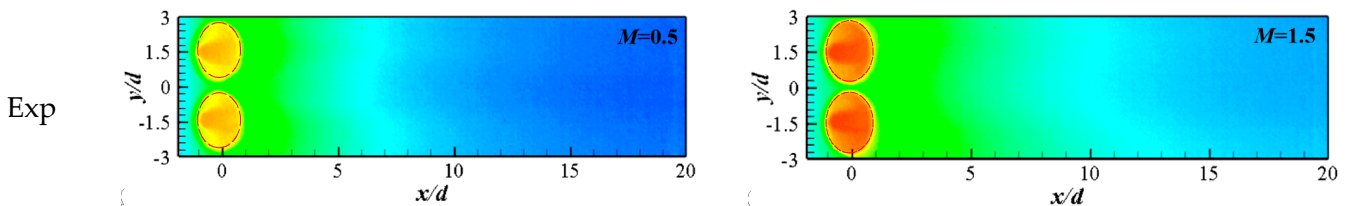

CFD
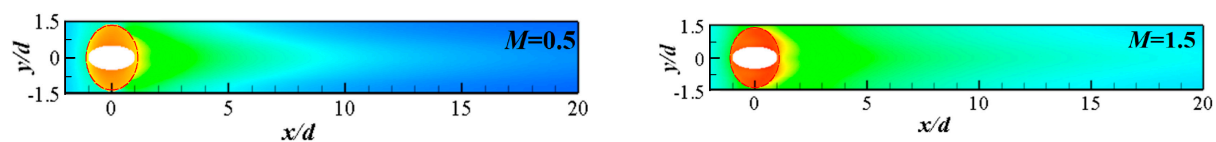

(c) Elliptic trench $\left(D_{\mathrm{x}}=2.2 d, D_{\mathrm{y}}=2.8 d, h=1 d\right)$

Figure 5. Distributions of overall cooling effectiveness on the cooling surface. 


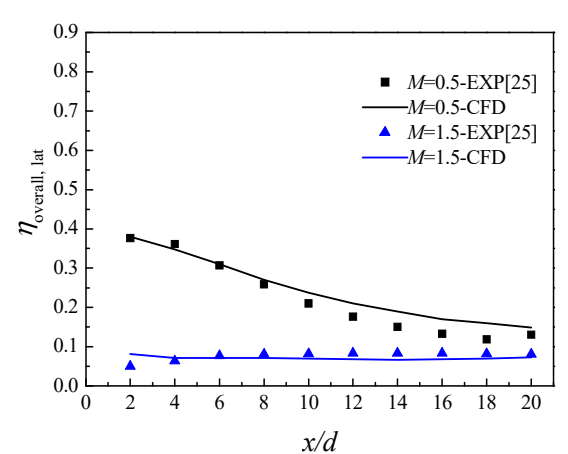

(a) Round hole

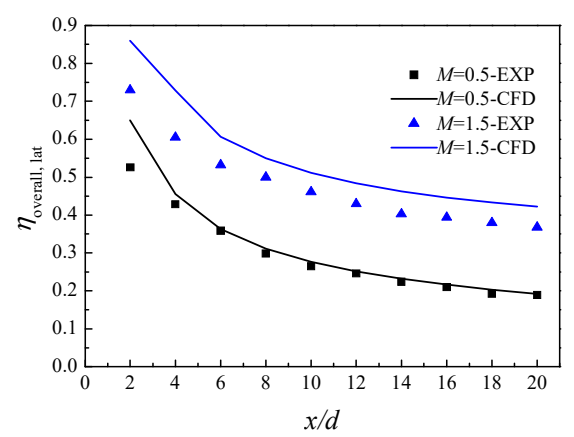

(c) W-shaped trench $\left(w=1.7 d, h=1 d, \alpha=60^{\circ}\right)$

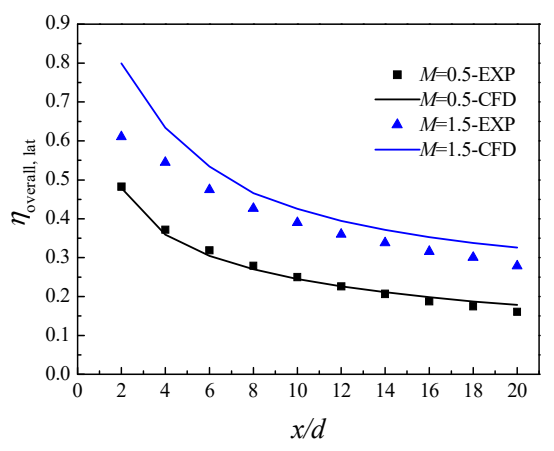

(b) Transverse trench $(w=3.0 d, h=1 d)$

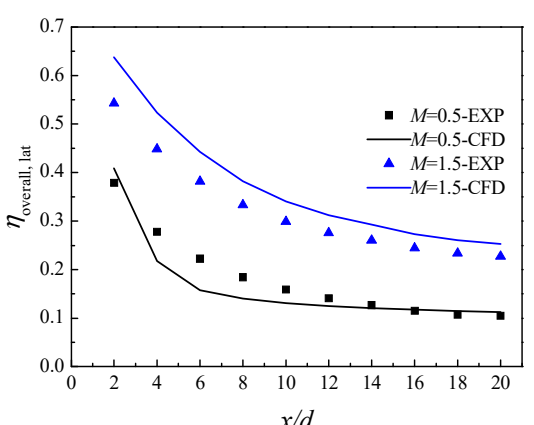

(d) Elliptic trench $\left(D_{\mathrm{x}}=2.2 d, D_{\mathrm{y}}=2.8 d, h=1 d\right)$

Figure 6. Quantitative comparison between CFD results and experimental results.

\section{CFD Results Analysis}

\subsection{Transverse Trench}

Figure 7 shows the variation of laterally averaged adiabatic cooling effectiveness $\left(\eta_{\text {ad,lat }}\right)$ with the streamwise distance $(x / d)$. For the round hole, $\eta_{\text {ad,lat }}$ shows a continued decline as $x / d$ increases at a low blowing ratio, but has a slight increase in the far field region due to the reattachment of a separated coolant jet at a high blowing ratio. For the transverse trench, $\eta_{\text {ad,lat }}$ decreases constantly with the increase of $x / d$ even at a high blowing ratio. The optimal blowing ratio for the round hole is about 0.5. However, for trench-film cooling in the present case, the optimal blowing ratio is between 1.0 1.5. Figure 8a,b show the streamline distributions for film cooling of the transverse trench and round hole at $M=1.5$, respectively, and the background color represents gas temperature. The most typical feature for trench film cooling is that a pair of recirculating vortices is formed inside the trench. The entrainment of recirculating vortices promotes the spreading of coolant in the lateral direction $[16,26]$. The existence of the trench also increases the actual jet exit area, reduces the actual jet velocity and mitigates the jet detachment downstream of the hole. In the flow fields downstream of the round hole, a pair of kidney vortices (also called countered rotating vortices) dominate the flow field and promote the mixture between mainstream and coolant [27]. For trench film cooling, beside kidney vortices, a pair of anti-kidney vortices can be observed. The anti-kidney vortices with the opposite rotating direction of kidney vortices mitigate the detachment of coolant jet and improve the film cooling performance [28]. Overall, compared with a round hole, a trench hole generates better cooling performance, especially at a high blowing ratio. Moreover, the results from Lu et al. [11-13] show that the heat transfer coefficient does not change obviously after introducing the trench. 


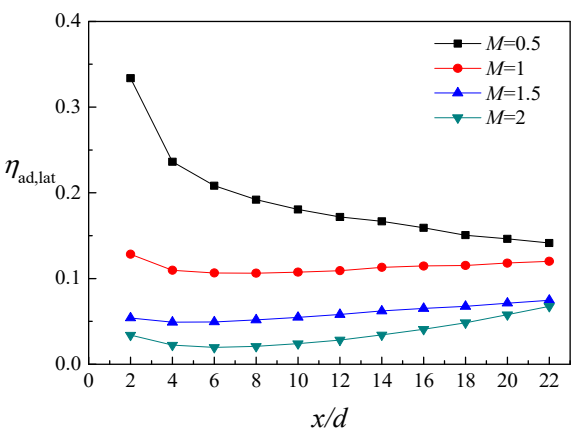

(a) Round hole

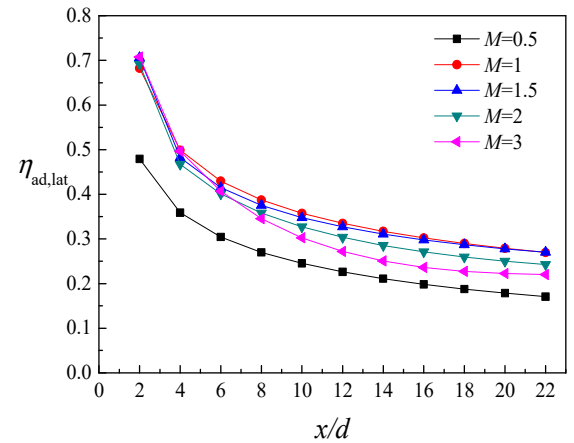

(b) Transverse trench $(w=3 d, h=0.75 d)$

Figure 7. Variation of laterally averaged adiabatic cooling effectiveness with streamwise distance.
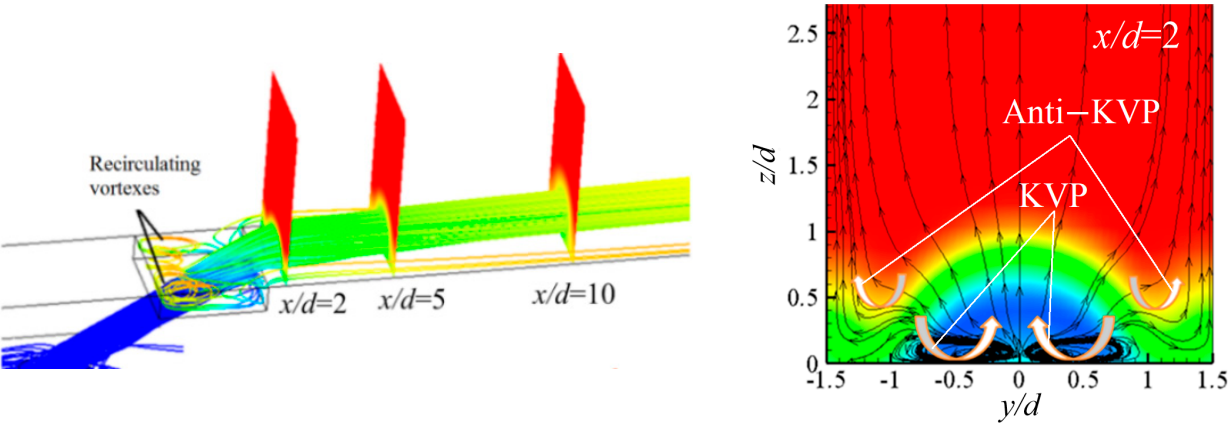

(a) Transverse trench $(w=3 d, h=0.75 d)$
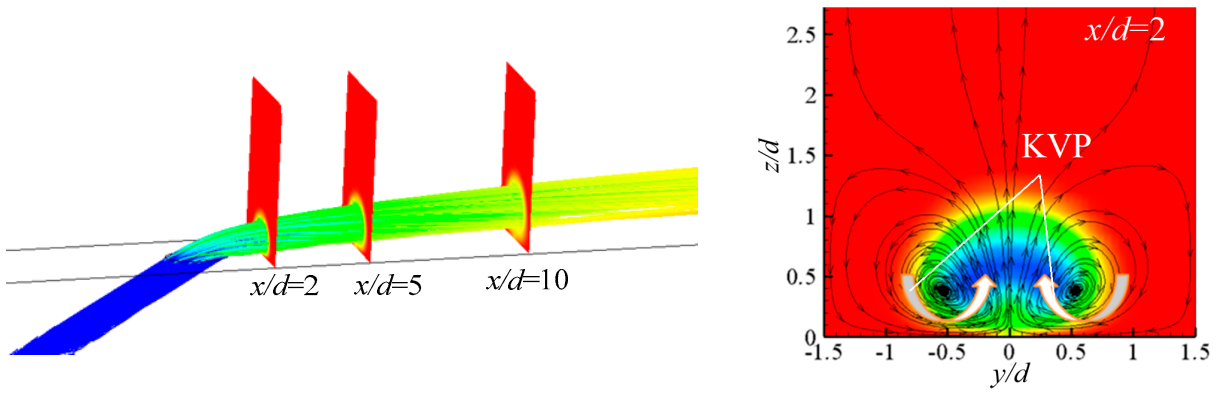

(b) Round hole

Figure 8. Streamline distributions for film cooling of round hole and transverse trench at $M=1.5$. (KVP: kidney vortex pair).

Figure 9 shows the variation of local adiabatic cooling effectiveness $\left(\eta_{\text {ad,loc }}\right)$ with the spanwise distance $(|y / d|)$. For the round hole, $\eta_{\text {ad,loc }}$ has the maximum value at $y / d=0$, and then decreases sharply with the increase of $|y / d| . \eta_{\text {ad,loc }}$ in the centerline region $(|y / d|<1)$ decreases by increasing the blowing ratio from 0.5 to 2.0 . However, the effects of the blowing ratio on $\eta_{\text {ad,loc }}$ at $|y / d|>1$ is unobvious. For the transverse trench, at a low blowing ratio $(M=0.5)$, the distribution of $\eta_{\mathrm{ad}, \mathrm{loc}}$ in the lateral direction is similar to that for the round hole. However, at a high blowing ratio, as the lateral distance increases, $\eta_{\text {ad,loc }}$ increases firstly $((|y / d|<0.5)$, then decreases $(0.5<|y / d|<1.2)$, and shows a slight increase finally $(|y / d|>1.2)$. Figure 10 shows the distributions of flow fields on the exit plane of holes; the background color represents the vertical velocity, and the arrow represents the clockwise or anti-clockwise rotation direction of the vortex. Compared with the transverse trench, the vertical velocity on the exit surface of the round hole distributes more uniformly and is of higher value. It results in the detachment of coolant jet immediately downstream of the round hole at a high blowing ratio. For the transverse trench, a pair of vortices is 
formed, and the entrainment of the vortex pair promotes the lateral spreading of coolant inside the trench. It results in the wavy distribution of vertical velocity in the lateral direction. The wave crest locates at $y / d=0.0$ where $\eta_{\text {ad, loc }}$ has a local minimum value at a high blowing ratio.

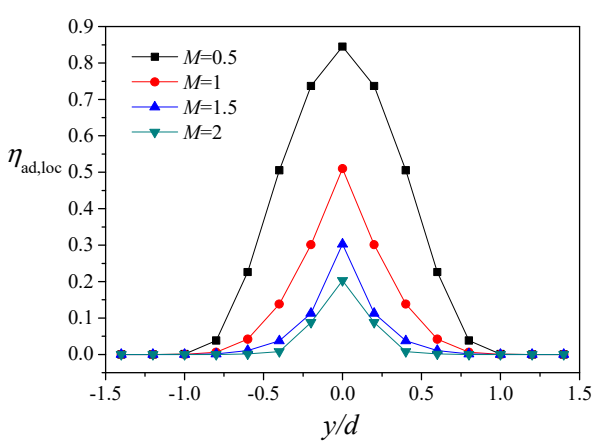

(a) Round hole

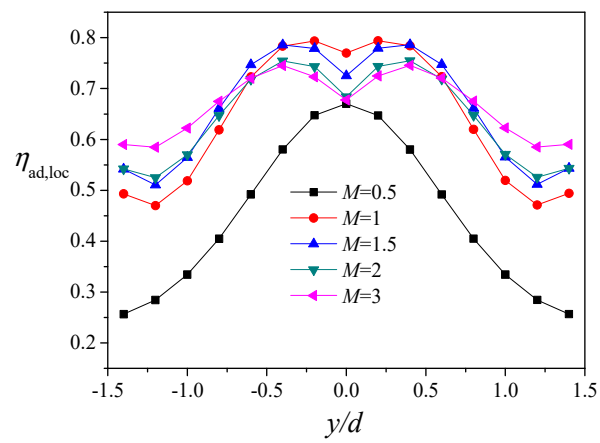

(b) Transverse trench $(w=3 d, h=0.75 d)$

Figure 9. Variation of local adiabatic film cooling effectiveness with spanwise distance on the cross section of $x / d=2.2$.

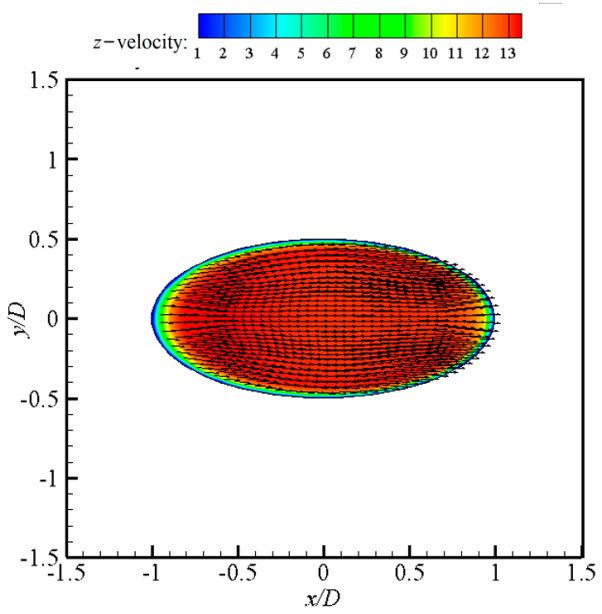

(a) Round hole

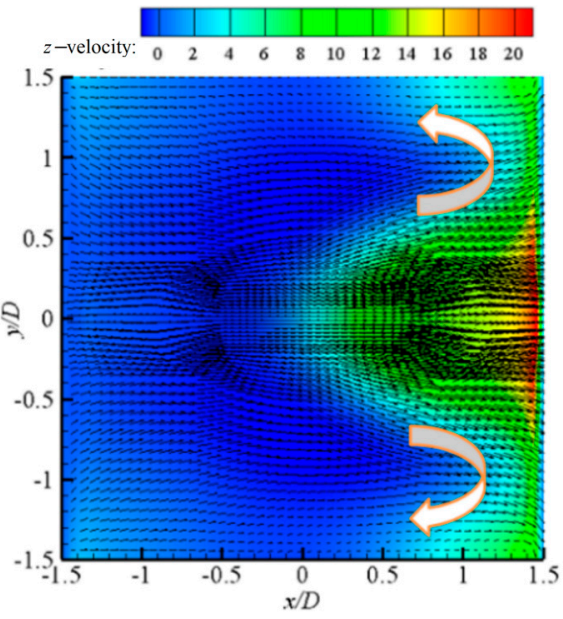

(b) Transverse trench $(w=3 d, h=0.75 d)$

Figure 10. Distribution of flow fields on the exit plane of the film cooling hole at $M=1.5$ (unit: $\mathrm{m} / \mathrm{s}$ ).

Figure 11a shows the effects of trench width $(W / d)$ and depth $(h / d)$ on the area-averaged adiabatic film cooling effectiveness $\left(\eta_{\mathrm{ad}, \mathrm{av}}, 2<x / d<20\right)$. The deep trench gives the highest $\eta_{\mathrm{ad}, \mathrm{av}}$, and $\eta_{\mathrm{ad}, \mathrm{av}}$ decreases with the decrease of trench depth. For a shallow trench, the coolant trajectory is hardly affected by the trench, and the pronounced flow separation still takes place downstream of the hole. Inside the deep trench, the recirculating flow reduces the coolant penetration into the mainstream and improves the coolant uniformity. Accordingly, the coolant ejected from the deep trench exhibits better covering performance on the cooling surface downstream of the trench. For most cases, the film cooling performance can be improved effectively by reducing the trench width. For a narrow trench, because of the effect of recirculating flow, the mainstream cannot enter the trench, which enhances the film cooling effectiveness. For a wide trench, due to the mainstream intursion, the recirculation of the coolant inside the trench is mitigated, which results in the decrease of cooling effectiveness [12,16]. Compared with trench width, the effect of trench depth on cooling effectiveness is more pronounced. Figure $11 \mathrm{~b}$ shows the effects of geometric parameters on the discharge coefficient $\left(C_{d}\right)$. The increase of $h / d$ reduces the mixing loss between the mainstream and coolant jet, and the decrease of $w / d$ results in the increase of the actual blowing ratio. Thus, $C_{d}$ increases with the increase of $h / d$ but the decrease of $w / d$. Overall, a narrower and deeper trench generates better film cooling performance. 


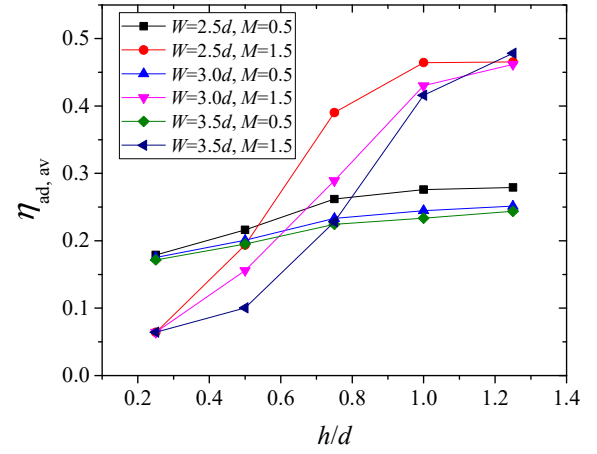

(a) Area-averaged adiabatic cooling effectiveness

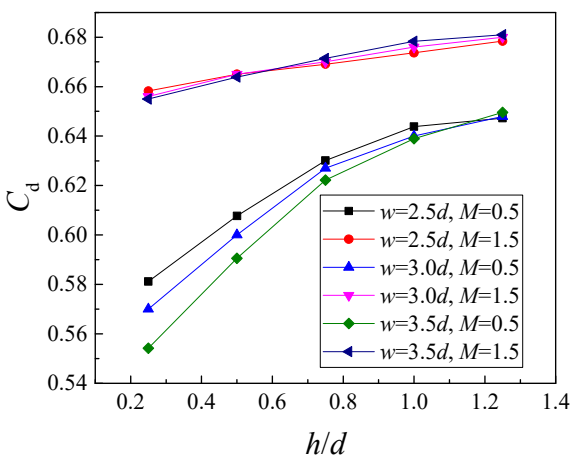

(b) Discharge coefficient

Figure 11. Effects of trench depth and width on film cooling performance for transverse trench.

\subsection{W-Shaped Trench}

Figure 12a shows the variation of laterally averaged adiabatic film cooling effectiveness with the streamwise distance. As the blowing ratio increases, $\eta_{\text {ad,lat }}$ increases firstly, and then decreases. The optimal blowing ratio for the $\mathrm{W}$-shaped trench is about 1.5. Compared with the transverse trench, the cooling performance for the $\mathrm{W}$-shaped trench is better. Figure $12 \mathrm{~b}$ shows the variation of local adiabatic film cooling effectiveness with the spanwise distance. At a low blowing ratio $(M=0.5)$, as $|y / d|$ increases, $\eta_{\text {ad,loc }}$ decreases firstly, and then increases. $\eta_{\text {ad,loc }}$ at $M=0.5$ has a maximum value at $y / d=0.0$ and a minimum value at $|y / d|=0.75$. At a moderate blowing ratio $(M=1.5), \eta_{\text {ad,loc }}$ decreases slightly as $|y / d|$ increases from 0.0 to 1.0 , and then shows a sharp decrease as $|y / d|$ exceeds 1.0. At a high blowing ratio $(M=3.0)$, the changing trend of $\eta_{\text {ad,loc }}$ is similar to that at a moderate blowing ratio. However, $\eta_{\mathrm{ad}, \mathrm{loc}}$ at a high blowing ratio has a local minimum value at $y / d=0.0$. Figure 13 shows the streamline distributions for film cooling of the $\mathrm{W}$-shaped trench, and the background color represents the gas temperature. At a low blowing ratio, pronounced recirculating flow can be observed inside the trench and promotes the lateral spreading of coolant. At a high blowing ratio, the coolant jet exhibits slight detachment in the region of $x / d=2.0$, but the coverage performance of coolant is still much better than the transverse trench and the round hole, especially in the far-field region $(x / d>5.0)$. Similar to the transverse trench, the kidney vortex pair and anti-kidney vortex pair exist simultaneously downstream of the $\mathrm{W}$-shaped trench. The anti-kidney vortices improve cooling performance, while the kidney vortices degrade cooling performance. However, compared with the transverse trench, the $\mathrm{W}$-shaped trench exhibits a stronger anti-kidney vortex pair and weaker kidney vortex pair.

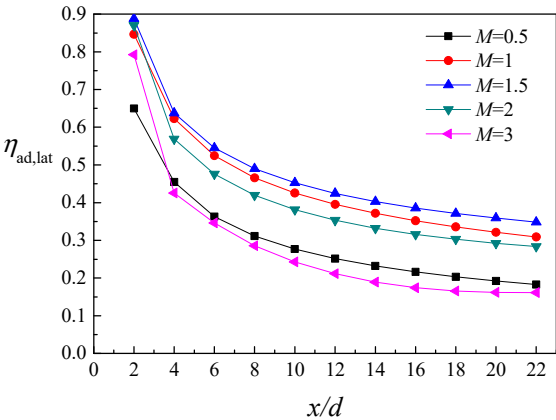

(a) Laterally averaged value

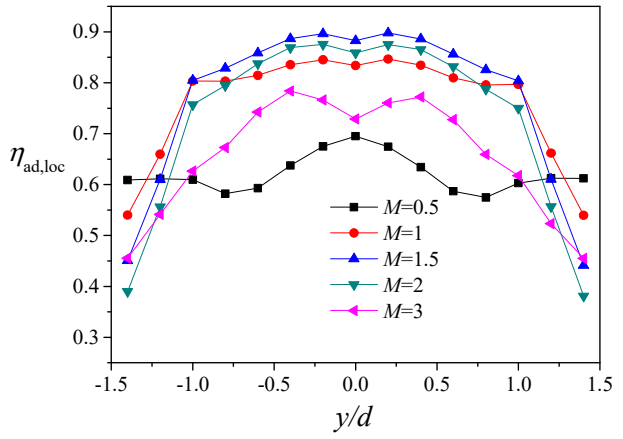

(b) Local value on the cross section of $x / d=2.2$

Figure 12. Distributions of adiabatic film cooling effectiveness in different directions for $\mathrm{W}$-shaped trench $\left(w=1.2 d, h=0.75 d, \alpha=60^{\circ}\right)$. 


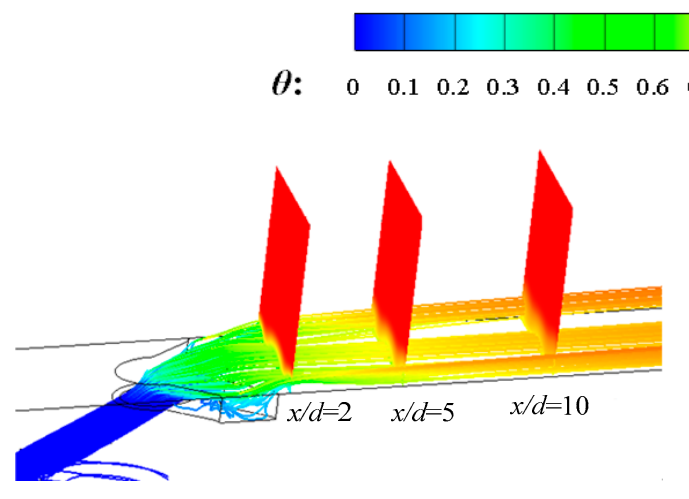

(a) $M=0.5$
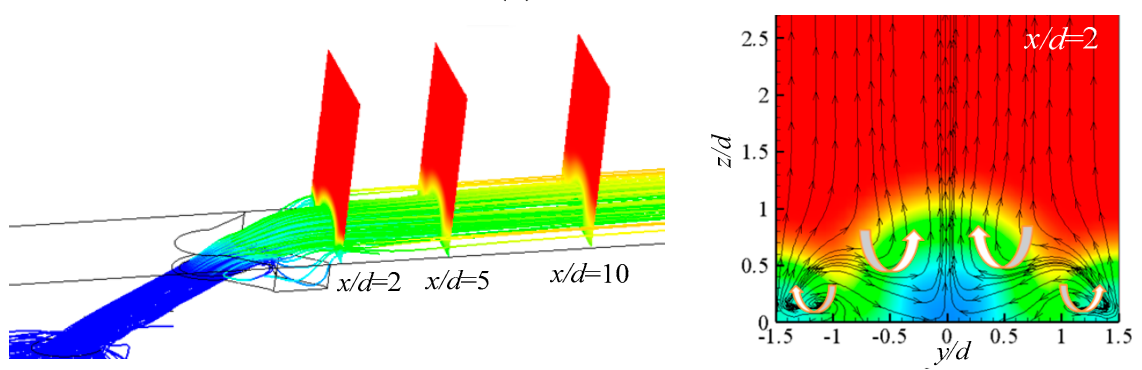

(b) $M=1.5$

Figure 13. Streamline distributions for film cooling of $\mathrm{W}$-shaped trench $\left(w=1.2 d, h=0.75 d, \alpha=60^{\circ}\right)$.

Figure 14a shows the effects of trench width and depth on the area-averaged adiabatic film cooling effectiveness $\left(\eta_{\mathrm{ad}, \mathrm{av}}, 2<x / d<20\right)$. For the narrow trench, because of the effect of recirculating flow, the mainstream cannot enter the trench, which enhances the film cooling effectiveness. For the wide trench, due to the mainstream intursion, the recirculation of the coolant inside the trench is mitigated, which results in the decrease of cooling effectiveness. Compared with trench width, the influence of $h / d$ on film cooling performance is much more obvious, especially at a high blowing ratio. At a high blowing ratio $(M=1.5), \eta_{\mathrm{ad}, \text { av }}$ increases from about 0.15 to 0.5 with the increase of $h / d$ from 0.25 to 1.3. At a low blowing ratio, $\eta_{\mathrm{ad}, \text { av }}$ also increases with the increase of trench depth, however, the variation amplitude is weaker compared to that at a high blowing ratio. Figure $14 \mathrm{~b}$ shows the effects of trench width and depth on the discharge coefficient. For a deep trench, the distribution of coolant velocity at the trench exit is uniform, and the area of the high speed zone is smaller compared with the shallow trench (shown in Figure 15), which reduces mixing loss between the mainstream and coolant jet. For the narrow trench, the jet velocity is higher, which results in the increase of flow loss. Thus, the increase of trench depth and width both cause the increase of the discharge coefficient.

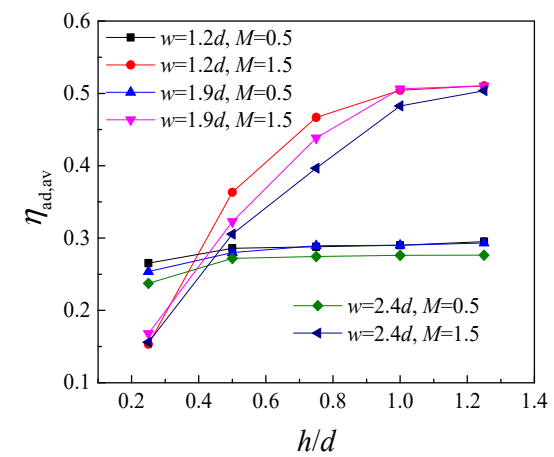

(a) Area-averaged adiabatic cooling effectiveness

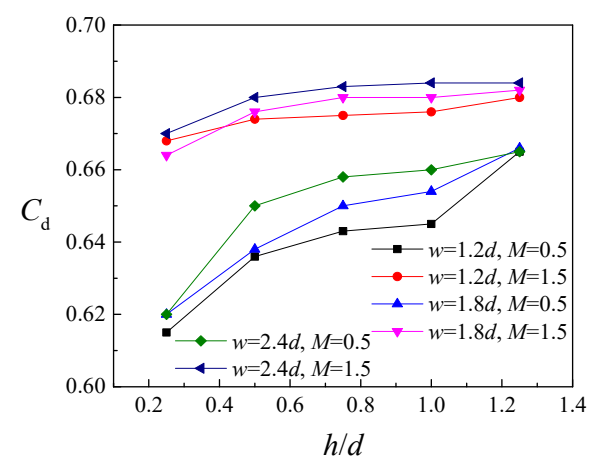

(b) Discharge coefficient

Figure 14. Effects of trench depth and width on film cooling performance for W-shaped trench $\left(\alpha=60^{\circ}\right)$. 


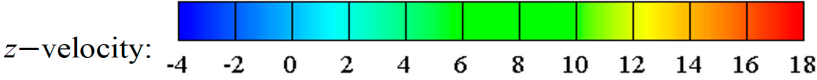

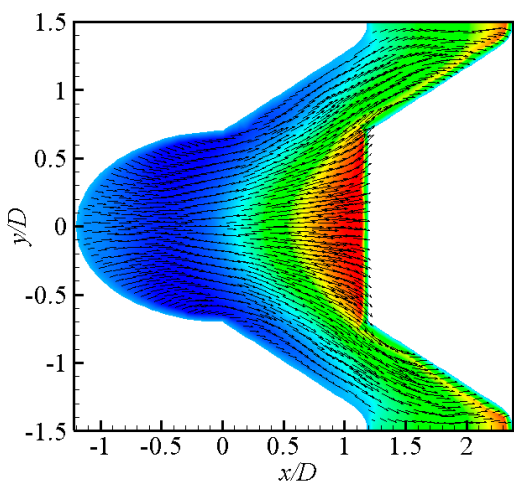

(a) $w=1.2 d, h=1.2 d, \alpha=60^{\circ}$

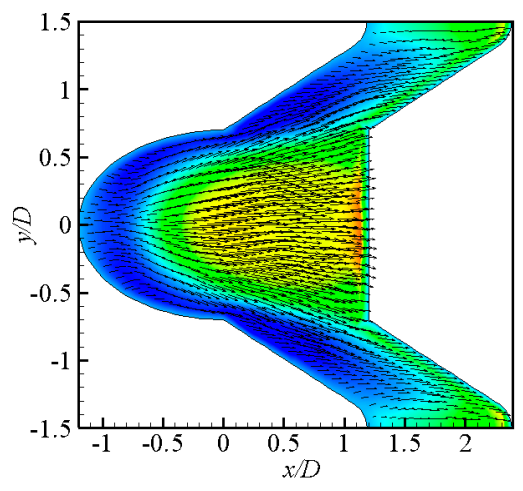

(b) $w=1.2 d, h=1.8 d, \alpha=60^{\circ}$

Figure 15. Distribution of flow fields on the exit plane of $\mathrm{W}-$ shaped trench at $M=1.5$ (Unit: $\mathrm{m} / \mathrm{s}$ ).

Figure 16a shows the effect of corner angle on the area-averaged adiabatic film cooling effectiveness. For a high corner angle, the lateral spreading inside the trench cannot be affected effectively by the $\mathrm{W}$-structure. In fact, as the corner angle approaches $180^{\circ}$, the W-shaped trench turns into a transverse trench, and the guide effect of the corner angle disappears. Conversely, a small corner angle promotes lateral spreading of coolant and improves distribution uniformity of the coolant velocity at the trench exit, which results in high cooling effectiveness. Figure $16 \mathrm{~b}$ shows the effect of corner angle on the discharge coefficient. $C_{d}$ decreases with the increase of the corner angle. It is because that high corner angle causes the decrease of the trench exit area, which results in high flow loss. However, because the changing interval of $\alpha$ is small $\left(40 \sim 60^{\circ}\right)$, the effects of the corner angle on the cooling effectiveness and discharge coefficient are not very obvious in the present study.

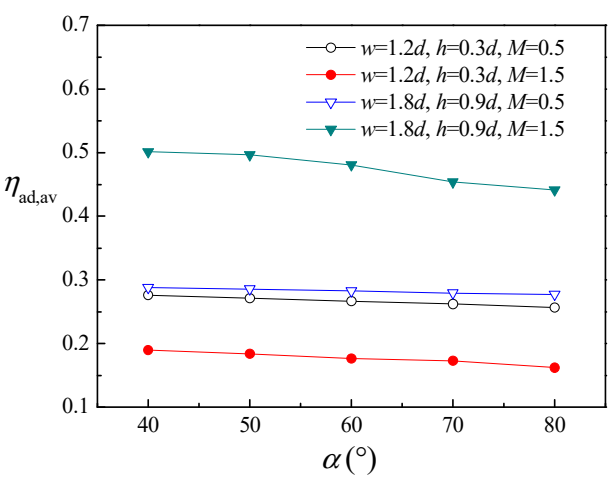

(a) Area-averaged adiabatic cooling effectiveness

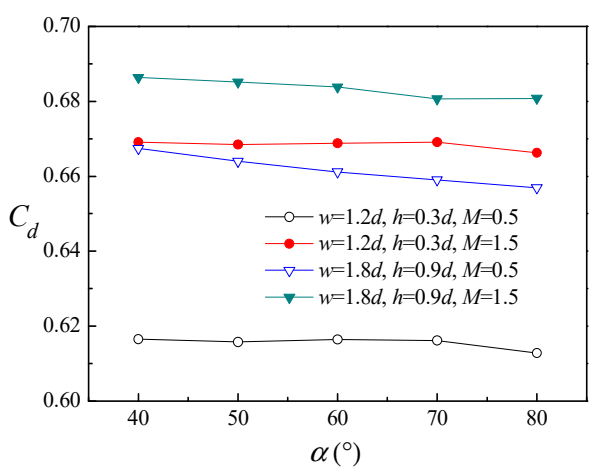

(b) Discharge coefficient

Figure 16. Effects of corner angle on film cooling performance for $\mathrm{W}$-shaped trench.

\subsection{Elliptical Trench}

Figure 17a shows the variation of laterally averaged adiabatic film cooling effectiveness with the streamwise distance. At a low blowing ratio, $\eta_{\text {ad,lat }}$ shows continued decrease with the increase of $x / d$. At a high blowing ratio, as $x / d$ increases, $\eta_{\text {ad,lat }}$ decreases firstly, and then increases. The rebound of $\eta_{\text {ad,lat }}$ in the far-field region can be attributed to the reattachment of the coolant jet. Figure $17 \mathrm{~b}$ shows the variation of local adiabatic film cooling effectiveness with the lateral distance. $\eta_{\text {ad,loc }}$ decreases with the increase of $|y / d|$ and has a maximum value at $y / d=0$. The changing trend of $\eta_{\text {ad,loc }}$ for the elliptical trench is similar to that for the round hole, but different from that for the transverse and W-shaped trench. Figure 18 shows the streamline distributions for film cooling for the elliptic trench, and the background color represents gas temperature. Compared with the transverse trench and the $\mathrm{W}$-shaped trench, the secondary flow inside the elliptic trench is 
unobvious, and lateral spreading of coolant is also weak in the elliptic trench. The kidney vortex pair takes the dominant role on the cross section downstream of the elliptic trench, while the anti-kidney vortex pair cannot be formed. The entrainment effect of the kidney vortex pair results in the detachment of coolant downstream of the hole and promotes the mixture between mainstream and coolant. Thus, the elliptic trench generates lower cooling effectiveness than the transverse and $\mathrm{W}$-shaped trench. However, the scale of the kidney vortex pair for the elliptic trench is smaller than that for the round hole, accordingly, the cooling effectiveness of the elliptic trench is higher than that with the round hole. In general, the flow characteristics for the elliptical trench are very similar to those for the round hole, but different from the transverse trench and $\mathrm{W}$-shaped trench.

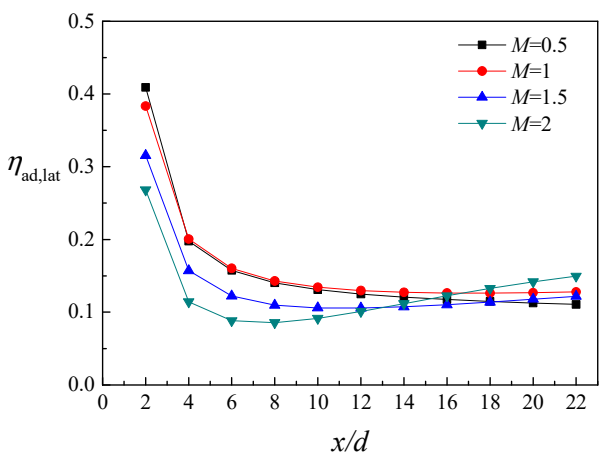

(a) Laterally averaged value

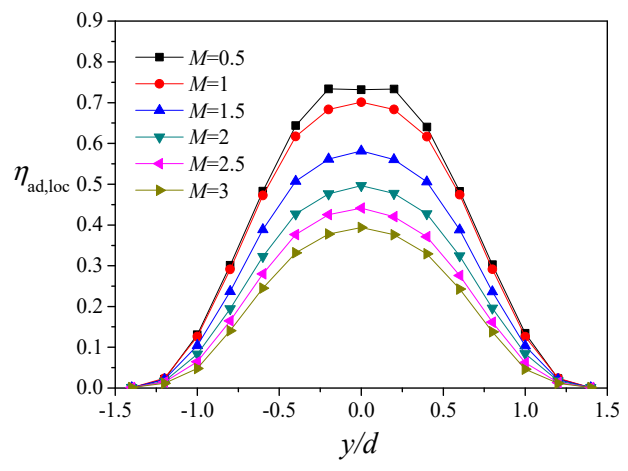

(b) Local value on the cross section of $x / d=2.2$

Figure 17. Distributions of adiabatic film cooling effectiveness for elliptic trench $\left(D_{\mathrm{x}}=2.4 d, D_{\mathrm{y}}=1.2 d\right.$, $h=0.75 d)$.

\section{$\begin{array}{lllllllllllll}\theta: & 0 & 0.1 & 0.2 & 0.3 & 0.4 & 0.5 & 0.6 & 0.7 & 0.8 & 0.9 & 1\end{array}$}
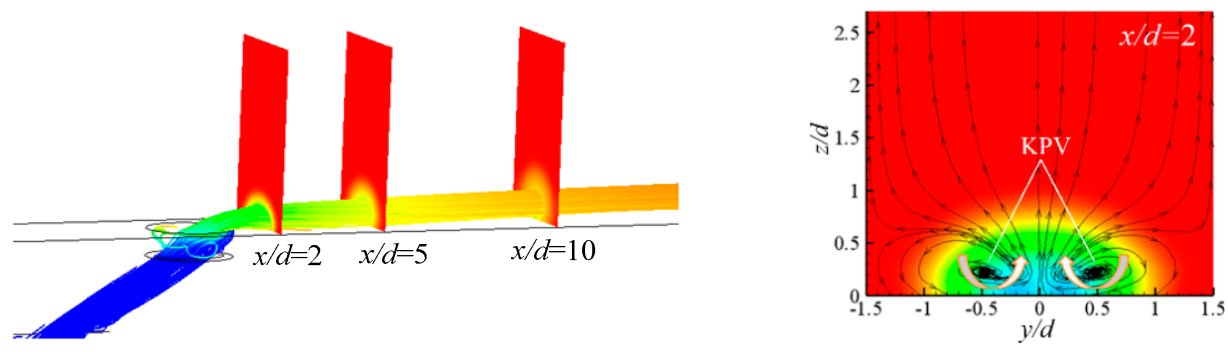

(a) $M=0.5$
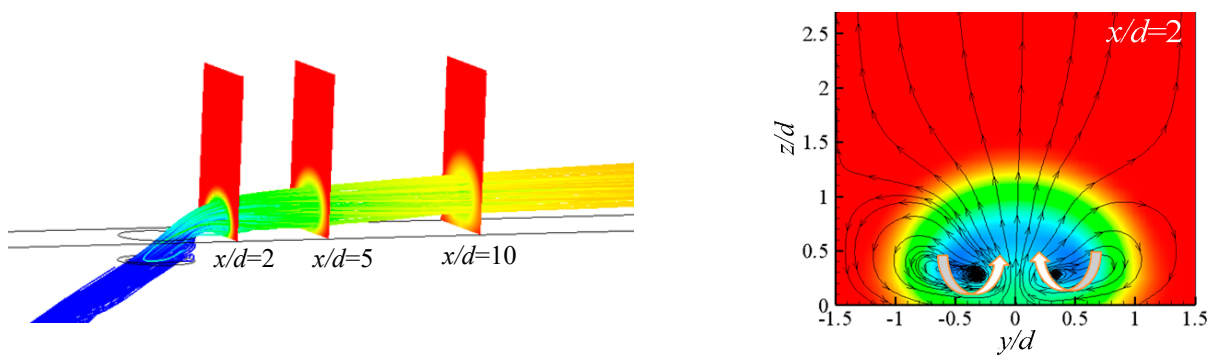

(b) $M=1.5$

Figure 18. Streamline distributions for film cooling of elliptic trench $\left(D_{\mathrm{x}}=2.4 d, D_{\mathrm{y}}=1.2 d, h=0.75 d\right)$.

Figure 19a shows the effects of axis length of the elliptical trench $\left(D_{\mathrm{x}}\right.$ and $\left.D_{\mathrm{y}}\right)$ on the area-averaged adiabatic film cooling effectiveness $\left(\eta_{\mathrm{ad}, \mathrm{av}}, 2<x / d<20\right)$. For small $D_{\mathrm{y}}$, $\eta_{\text {ad,av }}$ increases with the rise of $D_{\mathrm{x}}$. However, for large $D_{\mathrm{y}}$, this changing trend becomes wholly opposite, and $\eta_{\text {ad,av }}$ decreases with the increase of $D_{\mathrm{x}}$. It illustrates that there is an 
optimal exit area of the elliptical trench, and a too high and low exit area both deteriorate the cooling performance. If the exit area is higher than the optimal value, the actual jet velocity is too low, and the mainstream can penetrate into the trench. If the exit area is small, the actual jet velocity has high momentum, and shows a detachment effect from the wall downstream of the hole. Figure 20 shows the streamline distributions on the exit planes of elliptic trenches. Compared with the $\mathrm{W}$-shaped trench and transverse trench, the streamlines for the elliptic trench are smoother, and the recirculating vortex pair cannot be formed inside the trench. Figure $19 \mathrm{~b}$ shows the effects of axis length on discharge coefficient. The variation trend of $C_{\mathrm{d}}$ with $D_{\mathrm{x}}$ for large $D_{\mathrm{y}}$ is contrary to that for small $D_{\mathrm{y}}$. For $D_{\mathrm{y}}=2.5 d, C_{\mathrm{d}}$ increases with the increases of $D_{\mathrm{x}}$. However, for $D_{\mathrm{y}}=1.5 d, C_{\mathrm{d}}$ decreases as $D_{\mathrm{x}}$ increases. In general, as $D_{\mathrm{x}}$ is close to $D_{\mathrm{y}}$, the flow loss is relatively low.

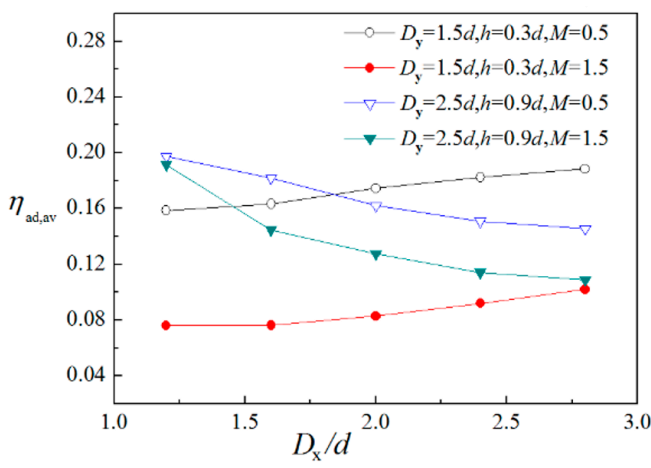

(a) Area-averaged adiabatic cooling effectiveness

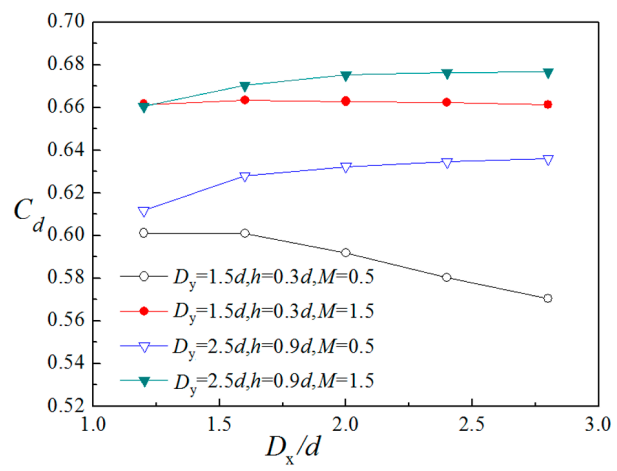

(b) Discharge coefficient

Figure 19. Effects of axis length on film cooling performance for elliptic trench.

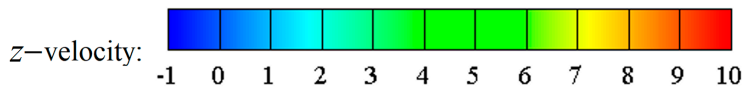

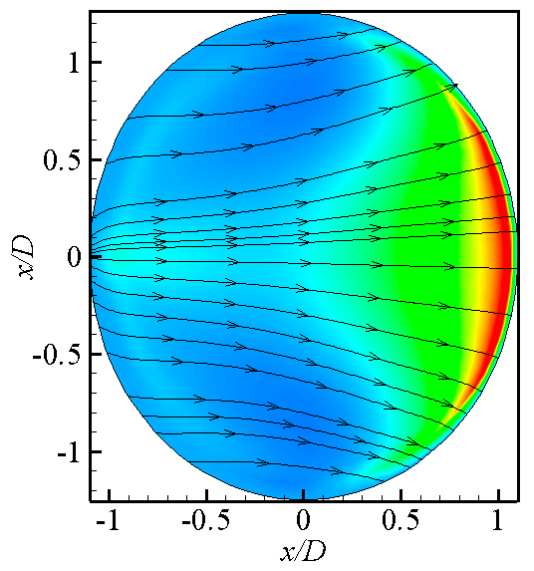

(a) $D_{\mathrm{x}}=2.2 d, D_{\mathrm{y}}=2.8 d, h=0.9 d$

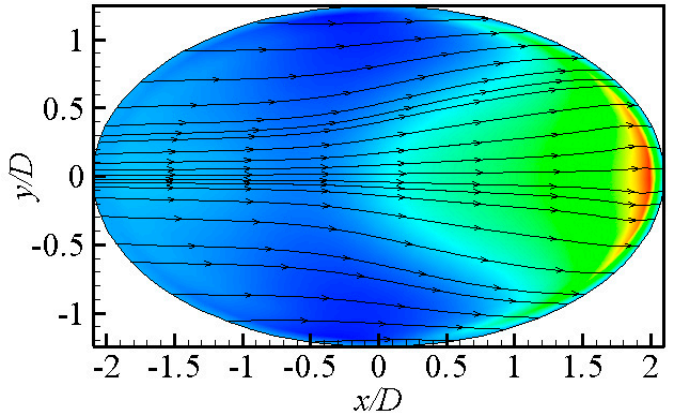

(b) $D_{\mathrm{x}}=3.2 \mathrm{~d}, D_{\mathrm{y}}=2.2 d, h=0.9 d$

Figure 20. Streamline distribution on the exit plane of elliptic trench at $M=0.5$ (Unit: $\mathrm{m} / \mathrm{s}$ ).

Figure 21 compares the distribution of adiabatic film cooling effectiveness on the wall for different trench shapes. At a low blowing ratio, the flow separation of the coolant downstream of these four kinds of holes is unobvious, and the cooling performance of the W-shaped trench is the best, while the cooling effectiveness of the round hole is the lowest. As the blowing ratio increases to 1.5, the coolant from the round hole and elliptic trench shows detachment from the wall, but the epileptic trench generates better cooling performance than the round hole. At this blowing ratio, the coverage performance of the $\mathrm{W}$-shaped trench is the best. At a very high blowing ratio, the coolant from these four kinds of holes exhibits detachment from the wall, however, the transverse trench and 
$\mathrm{W}$-shaped trench still generate high cooling effectiveness. In general, the $\mathrm{W}$-shaped trench generates the highest cooling effectiveness, while the cooling performance of the elliptic trench is the worst.

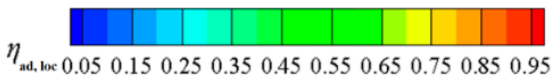

$M=0.5$
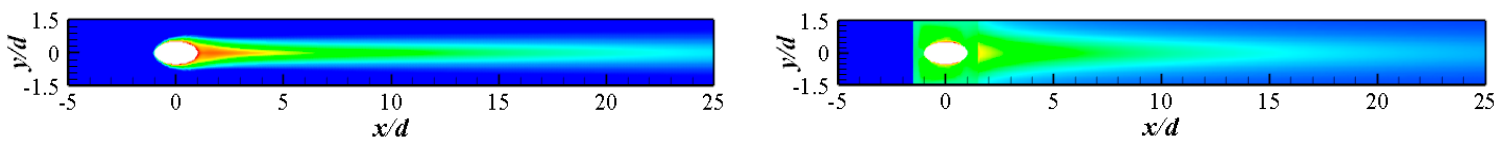

$M=1.5$
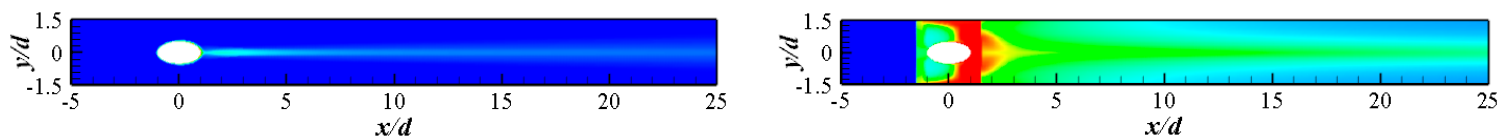

$M=2.5$
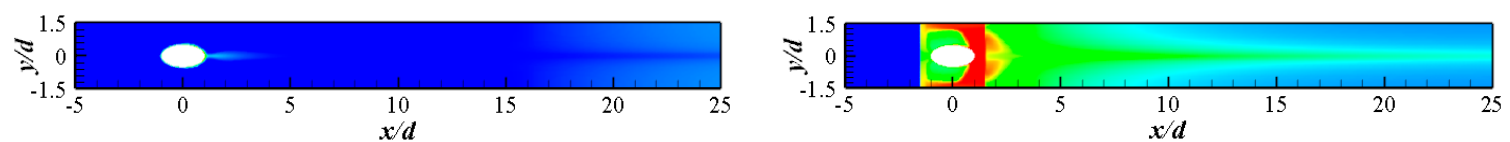

(a) Round hole

(b) Transverse trench $(w=3 d, h=0.75 d)$

$M=0.5$
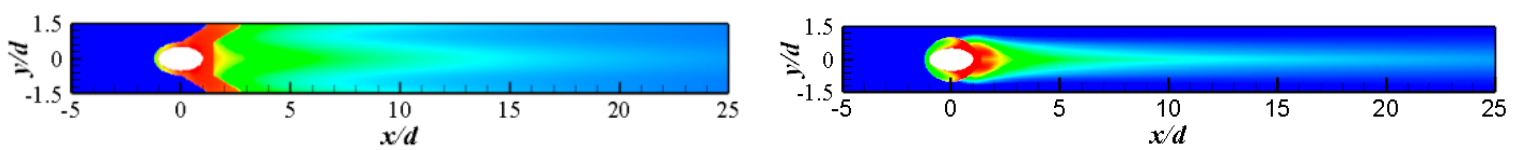

$M=1.5$
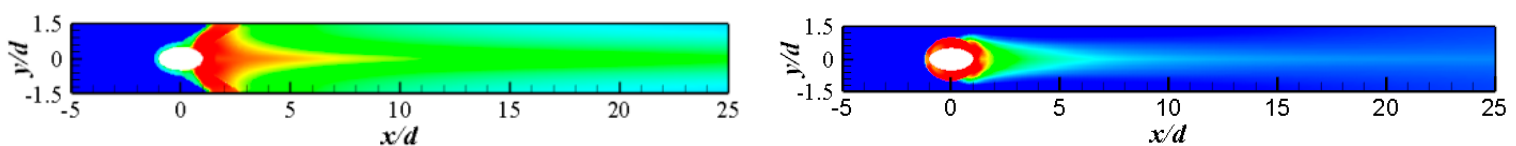

$M=2.5$
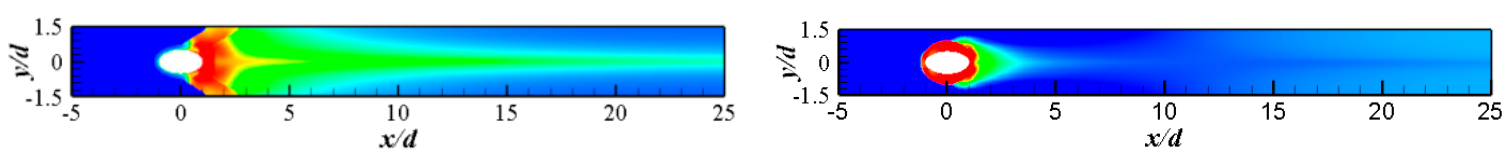

(c) W-shaped trench $\left(w=1.2 d, h=0.75 d, \alpha=60^{\circ}\right)$

(d) Elliptic trench $\left(D_{\mathrm{x}}=2.4 d, D_{\mathrm{y}}=2 d, h=0.75 d\right)$

Figure 21. Distribution of adiabatic film cooling effectiveness on the wall for different trench shapes.

\section{Conclusions}

The film cooling performances of the transverse trench, W-shaped trench and elliptic trench were investigated using the CFD method. The influences of blowing ratio and geometric parameters on the discharge coefficient and film cooling effectiveness were discussed. Some useful conclusions are listed below:

(1) Inside the transverse trench, a pair of recirculating vortices is formed, which promotes the lateral spreading of coolant. Downstream of the transverse trench, a kidney vortex pair and anti-vortex pair are formed simultaneously. The increase of trench depth and the decrease of trench width can both cause increases of film cooling effectiveness.

(2) Inside the W-shaped trench, the existence of a corner angle further promotes the coolant spreading in the lateral direction and generates higher film cooling effectiveness than the transverse trench. Similar to the transverse trench, the increase of trench depth and the decrease of trench width both result in the increase of cooling effectiveness. 
(3) For the elliptic trench, the flow characteristics are very similar to the round hole, and the kidney vortex pair is the largest-scale vortex structure. There exists an optimal exit area ratio (the exit area of elliptic trench/the exit area of round hole) for the elliptic trench, and too large or small an exit area ratio can lead to the degradation of film cooling effectiveness. The elliptic trench generates higher film cooling effectiveness than the round hole, but lower effectiveness than the transverse trench and W-shaped trench.

(4) As the blowing ratio increases, the discharge coefficient increases firstly and then keeps stable. The increase of trench depth and decrease of trench width results in the increase of the discharge coefficient for the transverse trench. For the W-shaped trench, the increase of the corner angle causes the decrease of the discharge coefficient. For the elliptic trench, the discharge coefficient increases with the decrease of the elliptic aspect ratio (major axis/minor axis).

Author Contributions: Conceptualization, C.W. and X.W.; methodology, X.W.; writing—original draft preparation, C.W.; writing—review and editing, X.D. and C.W.; funding acquisition, C.W. All authors have read and agreed to the published version of the manuscript.

Funding: This research was supported by the Fundamental Research Funds for the Central Universities (grant No: NS2020013) and National Science and Technology Major Project of China (grant No: 2017-III-0011-0037).

Institutional Review Board Statement: Not applicable.

Informed Consent Statement: Not applicable.

Data Availability Statement: Data available on request due to restrictions e.g., privacy or ethical.

Conflicts of Interest: The authors declare no conflict of interest.

\section{Nomenclature}

$A_{\mathrm{c}}$

$C_{\mathrm{d}}$

$d$

D

h

M

$m_{\mathrm{c}}$

$P^{*}$, in

$P_{\text {C,out }}$

$T$

$w$

$x, y$ and $z$

Greek symbols

$\alpha$

$\eta$

$\rho$

$\delta_{99}$

$\theta$

subscript

W

$\infty$

c

ad

av

loc

lat

overall

$x, y$ and $z$
Cross section area of round hole $\left(\mathrm{m}^{2}\right)$

Discharge coefficient (-)

Diameter of round hole ( $\mathrm{mm}$ )

Axis Length of elliptic crater ( $\mathrm{mm}$ )

Trench depth (mm)

Blowing ratio (-)

Coolant mass flow rate $(\mathrm{kg} / \mathrm{s})$

Inlet total pressure of secondary flow $(\mathrm{Pa})$

Static pressure downstream of trench $(\mathrm{Pa})$

Temperature (K)

Trench width (mm)

Streamwise, spanwise and vertical direction

Corner angle of $\mathrm{W}$-shaped trench $\left(^{\circ}\right)$

Cooling effectiveness (-)

Gas density $\left(\mathrm{kg} / \mathrm{m}^{3}\right)$

Boundary layer thickness of mainstream inlet (mm)

Dimensionless temperature $\left[=\left(T-T_{\mathrm{C}}\right) /\left(T_{\infty}-T_{\mathrm{C}}\right)\right]$

Wall

Mainstream

Coolant

At adiabatic condition

Area-averaged value

Local value

Laterally averaged value

At the condition considering heat conduction

Streamwise, spanwise and vertical component 


\section{References}

1. Bogard, D.G.; Thole, K.A. Gas turbine film cooling. J. Propuls. Power 2006, 22, 249-270. [CrossRef]

2. Bunker, R.S. A review of shaped hole turbine film-cooling technology. ASME J. Heat Transf. 2005, 127, 441-453. [CrossRef]

3. Bell, C.M.; Hamakawa, H.; Ligrani, P.M. Film cooling from shaped holes. ASME J. Heat Transf. 2000, 122, 224-232. [CrossRef]

4. Saumweber, C.; Schulz, A. Effect of geometry variations on the cooling performance of fan-shaped cooling holes. ASME J. Turbomach. 2012, 134, 061008. [CrossRef]

5. Wang, C.H.; Zhang, J.Z.; Zhou, J.H. Optimization of a fan-shaped hole to improve film cooling performance by RBF neural network and genetic algorithm. Aerosp. Sci. Technol. 2016, 58, 18-25. [CrossRef]

6. Kistenmacher, D.A.; Davidson, F.T.; Bogard, D.G. Realistic trench film cooling with a thermal barrier coating and deposition. ASME J. Turbomach. 2014, 136, 091002. [CrossRef]

7. Baheri, S.; Alavi Tabrizi, S.P.; Jubran, B.A. Film cooling effectiveness from trenched shaped and compound holes. Heat Mass Transf. 2008, 44, 989-998. [CrossRef]

8. Li, J.; Ren, J.; Jiang, H.D. Film cooling performance of the embedded holes in trenches with compound angles. In Proceedings of the ASME Turbo Expo 2010, Glasgow, Scotland, UK, 14-18 June 2010; pp. 1415-1424, ASME Paper No. GT2010-22337.

9. Bunker, R.S. Film cooling effectiveness due to discrete holes within a transverse surface slot. In Proceedings of the ASME Turbo Expo 2002, Amsterdam, The Netherlands, 3-6 June 2002; ASME Paper No. GT2002-30178.

10. Harrison, K.L.; Bogard, D.G. CFD predictions of film cooling adiabatic effectiveness for cylindrical holes embed in narrow and wide transverse trenches. In Proceedings of the ASME Turbo Expo 2007, Montreal, QC, Canada, 14-17 May 2007; ASME Paper No. GT2007-28005.

11. Lu, Y.; Nasir, H.; Ekkad, S.V. Film cooling from a row of holes embedded in transverse slots. In Proceedings of the ASME Turbo Expo 2005, Reno, NV, USA, 6-9 June 2005; ASME paper No. GT2005-68598.

12. Lu, Y.; Dhungel, A.; Ekkad, S.V.; Bunker, R.S. Effect of trench width and depth on film cooling from cylindrical holes embedded in trenches. ASME J. Turbomach. 2009, 131, 011003. [CrossRef]

13. Lu, Y.; Ekkad, S.V.; Bunker, R.S. Trench film cooling: Effect of trench downstream edge and hole spacing. In Proceedings of the ASME Turbo Expo 2008, Berlin, Germany, 9-13 June 2008; ASME Paper No. GT2008-50606.

14. Waye, S.K.; Bogard, D.G. High resolution film cooling effectiveness measurements of axial holes embedded in a transverse trench with various trench configurations. In Proceedings of the ASME Turbo Expo 2006, Barcelona, Spain, 8-11 May 2006; ASME Paper No. GT2006-90226.

15. Davidson, F.T.; KistenMacher, D.A.; Bogard, D.G. Film Cooling With a Thermal Barrier Coating: Round Holes, Craters, and Trenches. ASME J. Turbomach. 2014, 136, 041007. [CrossRef]

16. Lee, K.D.; Kim, K.Y. Film cooling performance of cylindrical holes embedded in a tranverse trench. Numer. Heat Transf. Part A Appl. 2014, 65, 127-143. [CrossRef]

17. Oguntade, H.I.; Andrews, G.E.; Burns, A.D.; Ingham, D.B.; Pourkashanian, M. Improved trench film cooling with shaped trench outlets. ASME J. Turbomach. 2013, 135, 021009. [CrossRef]

18. Lu, Y.; Dhungel, A.; Ekkad, S.V.; Bunker, R.S. Film cooling measurements for cratered cylindrical inclined holes. ASME J. Turbomach 2009, 131, 011005. [CrossRef]

19. Dorrington, J.R.; Bogard, D.G.; Bunker, R.S. Film Effectiveness Performance for Coolant Holes Imbedded in Various Shallow Trench and Crater Depressions. In Proceedings of the ASME Turbo Expo 2007, Montreal, QC, Canada, 14-17 May 2007; ASME Paper No. GT2007-27992.

20. Kross, B.; Pfitzner, M. Numerical and Experimental Investigation of the Film Cooling Effectiveness in a Novel Trench Configuration. In Proceedings of the ASME Turbo Expo 2012, Copenhagen, Denmark, 11-15 June 2012; ASME Paper No. GT2012-68125.

21. Wang, C.H.; Sun, X.K.; Fan, F.S.; Zhang, J.Z. Study on trench film cooling on turbine vane by large-eddy simulation. Numer. Heat Transf. Part A Appl. 2020, 78, 338-358. [CrossRef]

22. Wei, J.S.; Zhu, H.R.; Liu, C.L.; Song, H.; Liu, C.; Meng, T. Experimental study on the film cooling characteristics of the cylindrical holes embedded in sine-wave shaped trench. In Proceedings of the ASME Turbo Expo 2016, Seoul, Korea, 13-17 June 2016; ASME Paper No. GT2016-56856.

23. Zhang, B.L.; Zhang, L.; Zhu, H.R.; Wei, J.S.; Fu, Z.Y. Numerical study on the influence of trench width on film cooling characteristics of double-wave trench. In Proceedings of the ASME Turbo Expo 2017, Charlotte, VA, USA, 26-30 June 2017; ASME Paper No. GT2017-63552.

24. Fan, F.S. Large Eddy Simulation and Experimental Study on Film Cooling Flow and Heat Transfer Performance of Shallow Trenches. Master's Thesis, Nanjing Universtiy of Aeronautics and Astronautics, Nanjing, China, 2020.

25. Fan, F.S.; Wang, C.H.; Feng, H.K.; Zhang, J.Z. Large eddy simulation of film cooling from a shallow trench hole. J. Propuls. Technol. 2020, 41, 830-839.

26. Renze, P.; Schroder, W.; Meinke, M. Large eddy simulation of film cooling flow ejected in a shallow cavity. In Proceedings of the ASME Turbo Expo 2008, Berlin, Germany, 9-13 June 2008; ASME paper No. GT2008-50120.

27. Mahesh, K. The interaction of jets with crossflow. Annu. Rev. Fluid Mech. 2013, 45, 379-407. [CrossRef]

28. Haven, B.A.; Yamagata, D.K.; Kurosaka, M.; Yamawaki, S.; Maya, T. Anti-kidney pair of vortices in shaped holes and their influence on film cooling effectiveness. In Proceedings of the ASME Turbo Expo 2010, Glasgow, Scotland, 14-18 June 2010; ASME paper No. 97-GT-045. 